obtained, ease of diagnosis and the duration of tissue sampling and pathological reporting.

Results 108 participants were recruited, 57 male; mean age $66.9 \pm 10.9$. 85.2\% had a final diagnosis of malignancy. Median lesion size (IQR) was $25 \mathrm{~mm}$ (19-34.5). 62 (57.4\%) of lesions were in the head of pancreas. Tissue results from the FNB needle were significantly more accurate than FNA $(84.2 \%$ vs $75 \%, p=0.041)$ in discriminating malignant from benign masses. A greater proportion of FNB samples had abundant diagnostic material $(59.2 \%$ vs $44.4 \%, \mathrm{p}=0.017)$ and a straight forward diagnosis $(68.9 \%$ vs $51.9 \%, \mathrm{p}=0.03)$. Biopsy sampling time median (IQR) 685s (565-832) vs $752 \mathrm{~s}$ (651-835), $\mathrm{p}=0.0006)$ and pathology reporting times (191s (134-258) vs 332s (260-358), $\mathrm{p}<0.0001)$ were significantly shorter with FNB compared to FNA.

Conclusion The diagnostic performance of the SharkCore ${ }^{\mathrm{TM}}$ FNB needle was significantly better than that of a standard FNA needle in the diagnosis of solid pancreatic masses and was associated with better sample quality, ease of reporting and shorter sampling and pathological reporting times.

\section{PWE-073 EUS-GUIDED DRAINAGE OF PERIPANCREATIC FLUID COLLECTIONS: HOT AXIOS EXPERIENCE FROM A TERTIARY REFERRAL CENTRE}

\begin{abstract}
${ }^{1,2}$ Mohammad Farhad Peerally*, ${ }^{1}$ Fraser Goldie, ${ }^{1}$ Sophia Savva, ${ }^{1}$ Farooq Khan, ${ }^{1}$ Sudarshan Kadri. 'Digestive Diseases Centre, University Hospitals of Leicester NHS Trust, Leicester, UK; ${ }^{2}$ SAPPHIRE, Department of Health Sciences, University of Leicester, Leicester, UK
\end{abstract}

\subsection{6/gutjnl-2019-BSGAbstracts.304}

Introduction Endoscopic management of peripancreatic fluid collections (PFCs) has a high success rate and low mortality rate but plastic stents may be associated with high rates of blockage $(18 \%)$ while stent migration $(15 \%)$ remains an issue for tubular self-expanding metallic stents. ${ }^{1}$ The Hot-Axios (Boston Scientific) device offers a one-step combined diathermy-enabled access and deployment of a lumen-apposing self-expanding metal stent (LASEM). The aim of this study was to investigate the efficacy and safety of the Hot-Axios device for the management of patients with PFCs referred to a regional tertiary centre.

Methods This was a single-centre retrospective database study involving 27 consecutive patients who underwent drainage of PFCs using the Hot Axios device between 1stJanuary 2018 and 1st of January 2019. Data was obtained by interrogating GI reporting tool (Unisoft) and electronic health record systems, and is reported using simple descriptive statistics.

Results 28 procedures were performed in 27 patients (20 males (74\%), average age 57). 27 stents(96\%) were successfully placed. Clinical success rate was $89 \%$ (25 procedures, 2 increased in size due to complications, 1 had no change in size). 27(96\%) procedures were performed under conscious sedation (median doses: $3 \mathrm{mg}$ (range:1-6) Midazolam and 50 mcg of Fentanyl (range 25-100)). Stents remained in situ for an average of 55 days. $71 \%$ (20 procedures) required further washout (mean 1.4(1-5)). There was 1 perforation (4\%), 2 blocked stents (7\%), 2 migration (7\%). 30 day mortality was $7 \%$ (2 patients- due to pseudoaneursymal bleed unrelated to the procedure).

Conclusion EUS-guided management of PFCs using the HotAxios device offers high technical and clinical success rates, and low adverse event rates. Long term efficacy and safety data comparing plastic, conventional metal stents and LASEMs in a randomised controlled trial is required.

\section{REFERENCES}

1. Patil R, Ona MA, Papafragkakis C, Anand S, Duddempudi S. Endoscopic ultrasound-guided placement of AXIOS stent for drainage of pancreatic fluid collections. Annals of Gastroenterology: Quarterly Publication of the Hellenic Society of Gastroenterology. 2016;29(2):168.

\section{PWE-074 AMYLASE TESTING IN PRIMARY CARE - DOES IT IDENTIFY PATHOLOGY?}

Anju Phoolchund*, Fiona Vincent, Imogen Williams. Department of Gastroenterology, Poole Hospital NHS Foundation Trust

\subsection{6/gutjnl-2019-BSGAbstracts.305}

Introduction Serum amylase is a frequent requested biochemistry test in primary care. While useful in diagnosis of acute pancreatitis when significantly raised, it is not specific for this condition. it is often mildly raised in other GI and non-GI conditions, and can lead to unnecessary imaging and hospital referrals. We aimed to assess diagnostic value of serum amylase in primary care and its impact on secondary care services. Methods Data on patients in our local area who had serum amylase requested by their GP and processed at our laboratory during a 12-month period was assessed, comprising 2256 results. Children and repeat testing on single patients were excluded, with 2207 pancreatic amylase and 4 non-pancreatic amylase results reviewed. Indications, secondary care referrals and investigations, and final diagnoses were collected.

Results The commonest indication was abdominal pain (1123), followed by deranged LFTs (111) and "monitoring'or "routine test'(98). Over 150 separate indications were identified, including "anosmia'and "fertility check".

194 patients had one or more amylase result above normal range $(100 \mathrm{U} / \mathrm{L})$, with 9 patients between 209 and $299 \mathrm{U} / \mathrm{L}$, and 6 patients greater than $300 \mathrm{U} / \mathrm{L}$.

56 patients had their amylase repeated by the GP before decision was made about referrals, with 32 patients' results improving without treatment or investigation.

58 patients had an abdominal USS requested by their GP on the basis of raised amylase/symptoms as per indication of amylase request.

41 patients were referred to gastroenterology, 11 patients to the surgical team, and 3 to both teams. 12 of these were seen as " 2 week-wait'referrals.

132 of the 194 patients were found to have no pathology. Positive findings include pancreatic cancer (4), acute pancreatitis (4), cholelithiasis (11), choledocholithiasis requiring ERCP (2), macroamylasemia (1), raised salivary amylase (1). 3 further patients had been recently discharged by the surgical team with acute pancreatitis.

Of the 6 patients with serum amylase greater than $300 \mathrm{U} /$ L, 5 had a diagnosis of acute pancreatitis (3 were admitted shortly after blood tests, and 2 had been recently discharged from surgical wards).

Conclusions Diagnostic value of serum amylase is limited beyond the investigation of acute pancreatitis, when it should be a supportive test to a clinical diagnosis. When index of suspicion is high, these patients should be referred directly to hospital without waiting for outpatient results. While pathology including pancreatic malignancy was diagnosed in a small number of patients, it is unlikely that the mildly elevated 